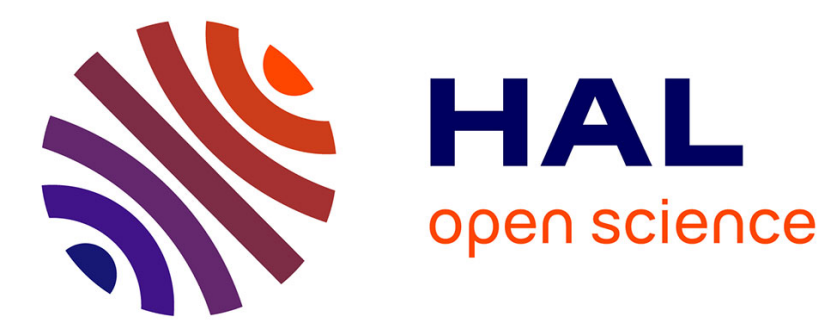

\title{
Raman wavelength conversion in a multipass cell
}

Nour Daher, Xavier Delen, Florent Guichard, Marc Hanna, Patrick Georges

\section{To cite this version:}

Nour Daher, Xavier Delen, Florent Guichard, Marc Hanna, Patrick Georges. Raman wavelength conversion in a multipass cell. Optics Letters, 2021, 46 (14), pp.3380-3383. 10.1364/OL.431675 . hal-03283155

\section{HAL Id: hal-03283155 \\ https://hal-iogs.archives-ouvertes.fr/hal-03283155}

Submitted on 9 Jul 2021

HAL is a multi-disciplinary open access archive for the deposit and dissemination of scientific research documents, whether they are published or not. The documents may come from teaching and research institutions in France or abroad, or from public or private research centers.
L'archive ouverte pluridisciplinaire HAL, est destinée au dépôt et à la diffusion de documents scientifiques de niveau recherche, publiés ou non, émanant des établissements d'enseignement et de recherche français ou étrangers, des laboratoires publics ou privés. 


\title{
Raman wavelength conversion in a multipass cell
}

\author{
Nour Daher,,${ }^{1,}{ }^{*}$ Xavier Délen, ${ }^{1}$ Florent Guichard, ${ }^{2}$ Marc Hanna, ${ }^{1}$ And \\ PATRICK GEORGES ${ }^{1}$.
}

${ }^{1}$ Université Paris-Saclay, Institut d'Optique Graduate School, CNRS, Laboratoire Charles Fabry, 91127, Palaiseau, France

${ }^{2}$ Amplitude, 11 Avenue de Canteranne, Cité de la Photonique, 33600 Pessac, France

*Corresponding author: nour.daher@institutoptique.fr

Received XX Month XXXX; revised XX Month, XXXX; accepted XX Month XXXX; posted XX Month XXXX (Doc. ID XXXXX); published XX Month XXXX

Positively chirped femtosecond pulses at $1030 \mathrm{~nm}$ are wavelength-converted using spontaneous and stimulated Raman scattering in a KGW crystal inserted inside a multipass cell. Recirculation in the cell and the Raman material allows both a high conversion efficiency and good spatial beam quality for the generated Stokes beams. The converted pulses can be compressed to subpicosecond duration. Multipass cells could be an appealing alternative to other Raman shifter implementations in terms of thermal effects, control of the Raman cascade, and overall output beam quality.

(C) 2021 Optical Society of America

http://dx.doi.org/10.1364/OL.99.09999

Nonlinear Raman frequency shifting is a widely used method to extend the wavelength coverage of laser sources in temporal regimes ranging from $\mathrm{CW}$ [1] to femtosecond pulses [2]. However, when the pump pulse duration is shorter than the Raman mode dephasing time, which is inversely proportional to the Raman gain bandwidth, stimulated Raman scattering (SRS)-induced frequency conversion is challenging due to the Raman transient regime, especially with femtosecond pump pulses. In this regime, the threshold pump intensity is increased compared with the steadystate case [3], and electronic third-order effects such as self-phase modulation (SPM), cross-phase modulation (XPM), and selffocusing can become predominant. These intensity-dependent effects can be alleviated by chirping the femtosecond input pump pulse. In this case, the generated Stokes pulses can be compressed since they partly inherit the chirp of the pump pulse [4]. This has been shown in single pass solid-state Raman crystals [5,6,2], fiber amplifiers [7], and high pressure gas-filled cells [4,8] and waveguides [9].

Performing SRS in solid-state media [10] is a robust, compact, and simple method to implement wavelength conversion since there is no controlled gas pressure or guiding structure. Several centimeters-long rods of Raman material are typically used in single or double pass, and power scaling can be limited by the onset of selffocusing effects [6]. Moreover, the converted output spatial beam quality is often degraded especially in single pass schemes where only a pump beam is irradiating the Raman medium while Stokes signals arise from noise [5]. However, it is possible to enhance the conversion efficiency and the spatial quality of the generated Stokes signals by providing a spatially coherent seed beam for the SRS process, through Raman amplification of supercontinuum pulses [2] or multistage Raman conversion schemes [11].

Recently, multipass cells (MPC) have been used to perform temporal compression of femtosecond laser pulses through the accumulation of SPM, with either solid $[12,13]$ or gaseous $[14,15]$ media as the nonlinear material. The distribution of nonlinearity over a large number of beam foci was shown to result in homogeneous and high quality output beams. When a solid material is used, the pulse peak power is not limited to the critical power of the material due to the recirculation in a bulk medium with a thickness well below the self-focusing distance [12,13].

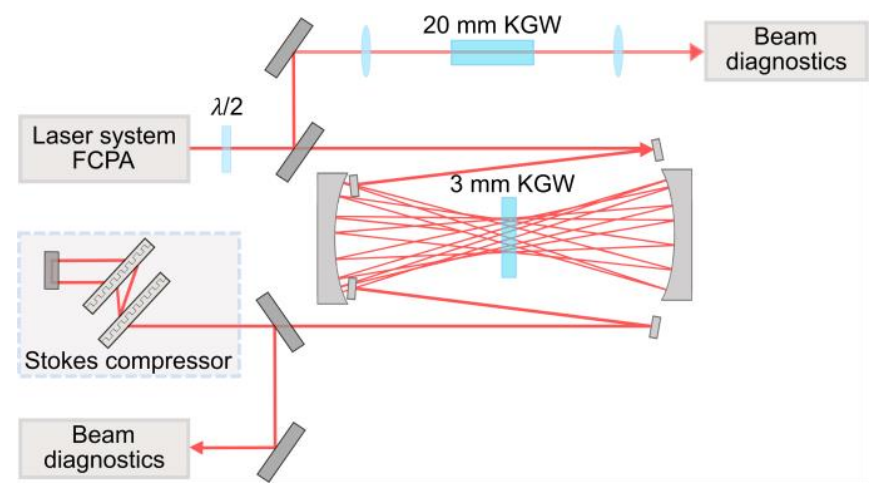

Fig. 1. Experimental setup.

In this work, we demonstrate the first Raman wavelength conversion inside a $3 \mathrm{~mm}$-long potassium gadolinium tungstate (KGW) crystal inserted in a MPC, in the stretched femtosecond pulse regime. Due to the large number of passes in the Raman medium provided by the MPC scheme, a large interaction length is achievable while operating at peak powers well above the critical power for self-focusing. In addition, co-propagation of the pump and Stokes beams inside the MPC leads to a spatial filtering effect that results in a high spatial quality of the converted beams. This 
property is illustrated with an experimental comparison with a single pass scheme in a $20 \mathrm{~mm}$-long KGW. The output beams in the MPC scheme are spatially and temporally characterized, demonstrating beam quality factors $\mathrm{M}^{2}<1.2$, compressibility down to subpicosecond durations, and conversion efficiencies of $44 \%$ and $30 \%$ for the first and second Stokes line for a linear polarization along the KGW Ng axis.
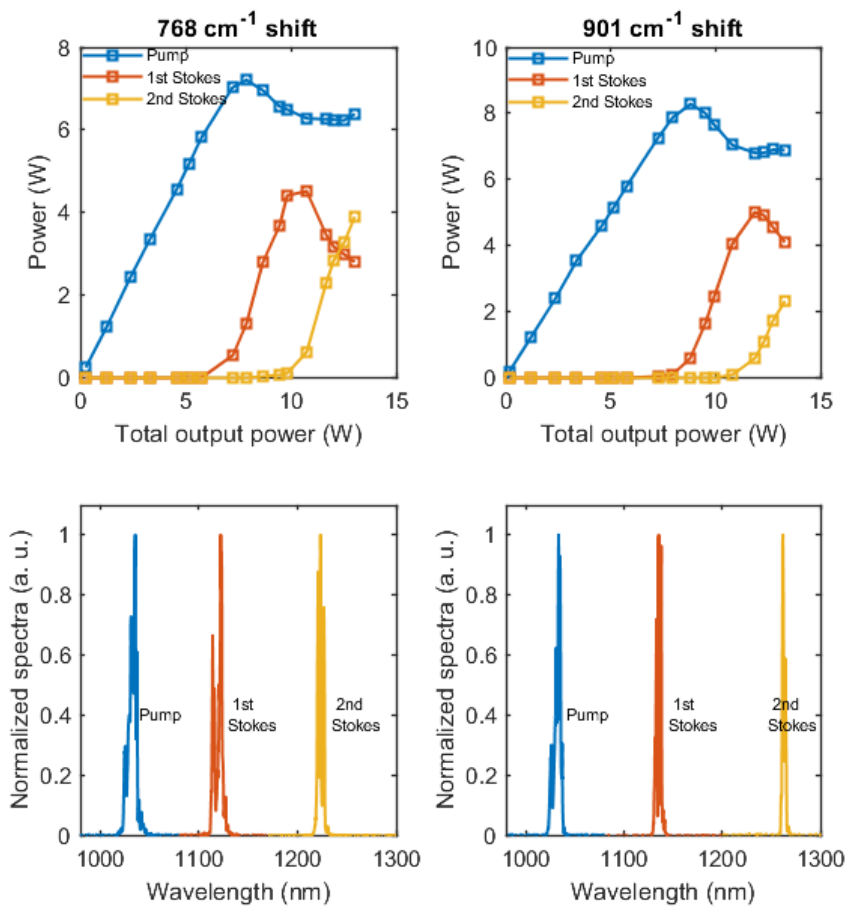

Fig. 2. Left column: (top) Average power of the pump and Stokes lines for the $768 \mathrm{~cm}^{-1}$ shift as a function of the total output power and (bottom) corresponding measured spectra; Right column: (top) Average power of the pump and Stokes lines for the $901 \mathrm{~cm}^{-1}$ shift as a function of the total output power and (bottom) corresponding measured spectra.

The scheme of our experimental setup is shown in Fig. 1. The input pulses are generated from a high-energy ytterbium-doped fiber chirped pulse amplifier (FCPA) delivering a maximum average power of $19 \mathrm{~W}$ with a $150 \mathrm{kHz}$ repetition rate, corresponding to 126 $\mu \mathrm{J}$ pulse energy, at $1030 \mathrm{~nm}$ central wavelength. The compressor of the FCPA is offset to positively chirp the $320 \mathrm{fs}$ pulses to obtain a duration of $18.7 \mathrm{ps}$, larger than the Raman dephasing times in KGW, of the order of a few ps [2]. The measured spatial quality factor of the laser beam is $\mathrm{M}^{2}=1.06$ in both horizontal and vertical directions. The pump beam is first directed to the MPC through an arrangement of three lenses in order to match the stationary beam of the MPC, resulting in a beam diameter of $\sim 400 \mu \mathrm{m}$ at the waist. The MPC is a Herriot cell [16] and consists of two concave mirrors with a radius of curvature of $300 \mathrm{~mm}$ separated by $504 \mathrm{~mm}$ and coated with a low group velocity dispersion dielectric stacks on a 1000-1300 $\mathrm{nm}$ bandwidth, allowing a high reflectivity of $>99.95 \%$ at the pump and both the first and second Stokes wavelengths. A 3 mm-thick anti-reflection coated KGW crystal with an aperture of 25 $\mathrm{mm} \times 25 \mathrm{~mm}$ is added near the center of the MPC as a nonlinear
Raman medium. The number of roundtrips in the MPC is 18, leading to a total propagation distance of $10.8 \mathrm{~cm}$ in KGW. The transmission of the MPC in linear regime, including the KGW crystal, is $80 \%$. The KGW crystal is chosen due to its high Raman gain, high damage threshold, and moderate thermal conductivity. It is cut normal to the Np axis, so one can obtain Raman shifts of $768 \mathrm{~cm}^{-1}$ and $901 \mathrm{~cm}$ ${ }^{1}$ when the input laser polarization is aligned with the $\mathrm{Ng}$ and $\mathrm{Nm}$ axes respectively [2]. Access to both Raman shifts in the experiment is allowed by using a half-wave plate placed before the MPC setup. At maximum input power, the estimated peak power is $6 \mathrm{MW}$, which is much higher than the critical power in the KGW of around $400 \mathrm{~kW}$. The self-focusing distance in these conditions is estimated to be $6.7 \mathrm{~cm}, 20$ times longer than the crystal length. The beam exiting the MPC is collimated with a $750 \mathrm{~mm}$-focal length lens and sent to various devices for characterization.
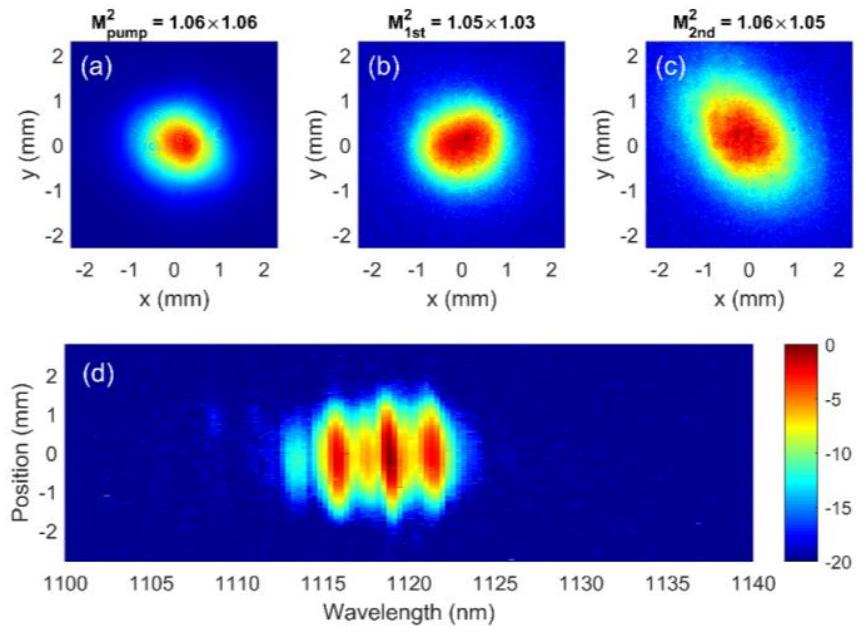

Fig. 3. Measured spatial beam profile of the (a) pump, (b) 1st Stokes and (c) $2^{\text {nd }}$ Stokes lines at the MPC output; (d) Experimental spectro-imaging measurement in logarithmic $(\mathrm{dB})$ scale.

Fig. 2 displays the average output power of the pump and Stokes lines as a function of the total power at the MPC output as well as the obtained spectra at maximum input power. For the 768 $\mathrm{cm}^{-1}$ shift, a maximum conversion efficiency of $44.6 \%$ and $30 \%$ is obtained at an input energy of $93 \mu \mathrm{J}$ and $126 \mu \mathrm{J}$ with spectra centered at $1120 \mathrm{~nm}$ and $1220 \mathrm{~nm}$ for the $1^{\text {st }}$ and $2^{\text {nd }}$ Stokes lines respectively. The maximum conversion efficiency drops to $42 \%$ and $17.4 \%$ for the $901 \mathrm{~cm}^{-1}$ shift corresponding to an input energy of $112 \mu \mathrm{J}$ and $126 \mu \mathrm{J}$, with spectra centered at $1135 \mathrm{~nm}$ and 1263 $\mathrm{nm}$ for the $1^{\text {st }}$ and $2^{\text {nd }}$ Stokes lines respectively. This difference in the conversion efficiencies is explained by the fact that the steady-state Raman gain coefficient is higher for the $768 \mathrm{~cm}^{-1}$ line than for the $901 \mathrm{~cm}^{-1}$ line.

In the following, we focus on the $768 \mathrm{~cm}^{-1}$ Stokes lines for further spatial and temporal characterizations. The spatial profiles measured at the output of the MPC for the pump at low power and for the Stokes lines at maximum conversion efficiency are shown in Fig. 3. A measured beam diameter of 2.3 by $2.4 \mathrm{~mm}, 2.5$ by $2.6 \mathrm{~mm}$, and 2.6 by $3.9 \mathrm{~mm}$ at $1 / \mathrm{e}^{2}$ is obtained at the MPC output for the pump, $1^{\text {st }}$ Stokes and $2^{\text {nd }}$ Stokes, respectively. This increase in beam size is coherent with the fact that a larger central wavelength translates into faster divergence for a given waist size. The reason 
for the larger ellipticity of the $2^{\text {nd }}$ Stokes beam is unclear, and it is not observed for the $2^{\text {nd }}$ Stokes beam of the $901 \mathrm{~cm}^{-1}$ shift. The $\mathrm{M}^{2}$ beam quality factors are measured by acquiring the beam profiles using an InGaAs camera translated through a focus. The measured beam quality factor of the $1^{\text {st }}$ Stokes and $2^{\text {nd }}$ Stokes lines is $\mathrm{M}^{2}=1.05 \times 1.03$ and $\mathrm{M}^{2}=1.06 \times 1.05$, respectively. This shows the preservation of the beam quality of the pump beam in the generated Stokes signals with a more pronounced ellipticity observed in the $2^{\text {nd }}$ Stokes. A spatio-spectral measurement is performed for the $1^{\text {st }}$ Stokes line by using an imaging spectrometer setup. It consists of an $\mathrm{f}-\mathrm{f}$ arrangement composed of an 800 lines/mm grating, a $100 \mathrm{~mm}$ focal length cylindrical lens and an InGaAs camera with a $30 \mu \mathrm{m}$ pixel size. The result, presented in Fig. 3(d), shows no spectral inhomogeneity in the beam.

The spatial properties of the Stokes beams are remarkable compared to previously reported single pass Raman conversion experiments, we therefore perform an experimental comparison to check this result. The same input chirped pump pulses are focused into a $20 \mathrm{~mm}$-long KGW crystal with a $400 \mathrm{~mm}$-focal length lens (upper part of Fig. 1). The KGW crystal is positioned slightly after the waist at a location where the beam diameter is $\sim 400 \mu \mathrm{m}$, to obtain a comparable intensity compared to the MPC case. The beam is then collimated and sent for characterization.
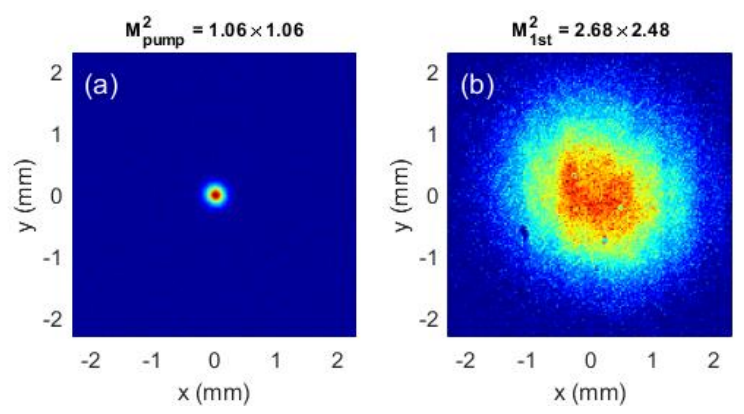

Fig. 4. Measured spatial profiles of the (a) pump and (b) $1^{\text {st }}$ Stokes line at the same position after collimation at the output of the $20 \mathrm{~mm} \mathrm{KGW}$ crystal.

Only the first Stokes line is observed at full pump pulse energy, with a converted power of $2 \mathrm{~W}$, corresponding to a conversion efficiency of $10 \%$. The measured beam profiles of the pump at low power and $1^{\text {st }}$ Stokes at maximum power are measured at the same position after collimation, and shown in Fig. 4. The diameter of the obtained Stokes beam is 3.3 by $3.2 \mathrm{~mm}$ at $1 / \mathrm{e}^{2}$, much larger than the measured diameter of the pump beam, of $0.4 \mathrm{~mm}$. The beam quality factor of the Stokes beam is $\mathrm{M}^{2}=2.7 \times 2.5$ along the horizontal and vertical directions, respectively. These spatial features can be interpreted as follows. The local Raman gain coefficient is proportional to intensity, which leads to a much larger gain at the beam center. As a result, for small enough pump depletion, the Raman converted beam in single pass is much smaller than the pump beam, which translates to a much larger beam after diffraction and collimation. In addition, for non-seeded setups, the process starts from quantum noise, so that the spatial coherence is low, resulting in mediocre beam quality factors. In contrast, in the MPC setup, the generated Stokes beam in the first passes is then copropagated with the pump, and spatial filtering imparted by the Raman gain at each pass progressively induces spatial coherence and a beam size that is comparable to the pump beam. Although this process reduces the Stokes signal growth compared to a single pass setup, this is compensated by the fact that multiple passes in the cell result in a larger interaction distance with the nonlinear material.
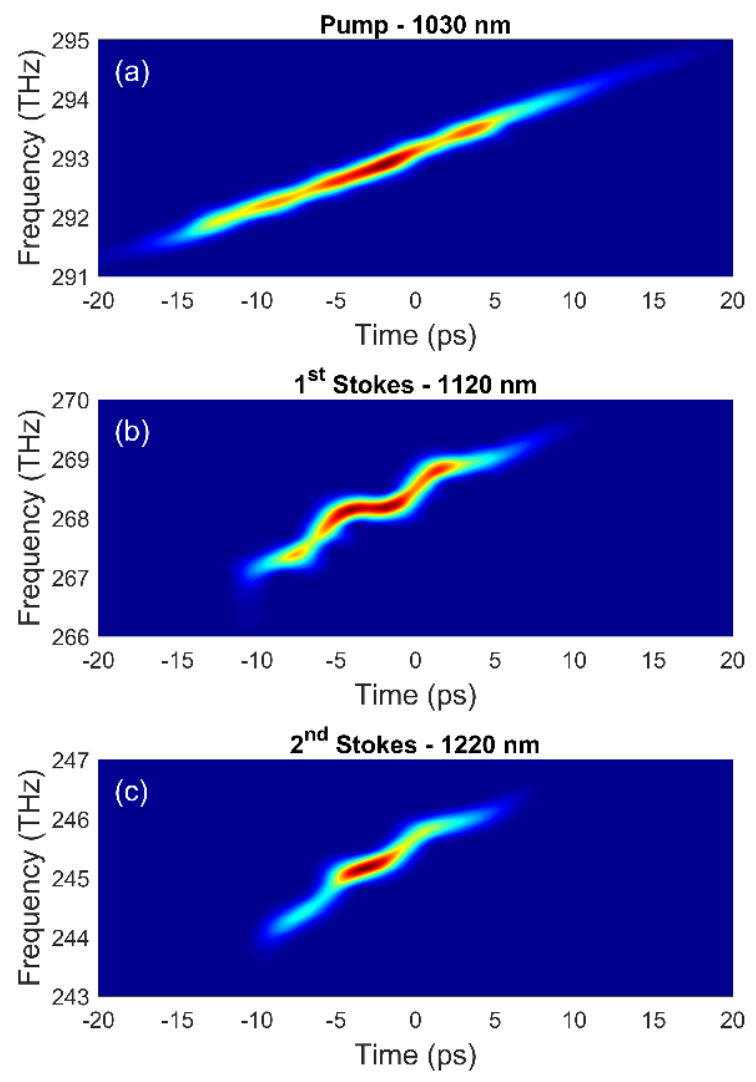

Fig. 5. Spectrogram of the (a) chirped input pump, non-compressed (b) $1^{\text {st }}$ Stokes and (c) $2^{\text {nd }}$ Stokes at the MPC output.

We now examine the temporal and spectral properties of the wavelength-converted beams at the output of the MPC setup. The pulses are characterized with a home-built second harmonicgeneration frequency resolved optical gating (FROG) setup, at maximum conversion efficiency for each of the Stokes lines. The obtained pulse duration at FWHM is $8.8 \mathrm{ps}$ and $6.3 \mathrm{ps}$ for the $1^{\text {st }}$ and $2^{\text {nd }}$ Stokes lines, respectively. This is because the delayed Raman response induces more efficient conversion the trailing edge of the input pulse, which results in shorter converted pulsewidths. Spectrograms obtained from the FROG-retrieved fields are plotted in Fig. 5 for the initial pump pulse, $1^{\text {st }}$ Stokes beam, and $2^{\text {nd }}$ Stokes beam. In the three cases, the instantaneous frequency increases over time with the same average slope, confirming that the input chirp is imprinted onto the converted pulses. However, we notice a staircase-like structure in the instantaneous frequency of the generated $1^{\text {st }}$ and $2^{\text {nd }}$ Stokes which has not been reported before to our knowledge. Although we do not have a definite explanation at this time, this characteristic could be related to the fact that the input chirped pump pulse does not exhibit a smooth temporal intensity profile. SPM and XPM effects can then act on the spectrograms as instantaneous frequency shifters, imparting these frequency oscillations around the main chirp slope. 

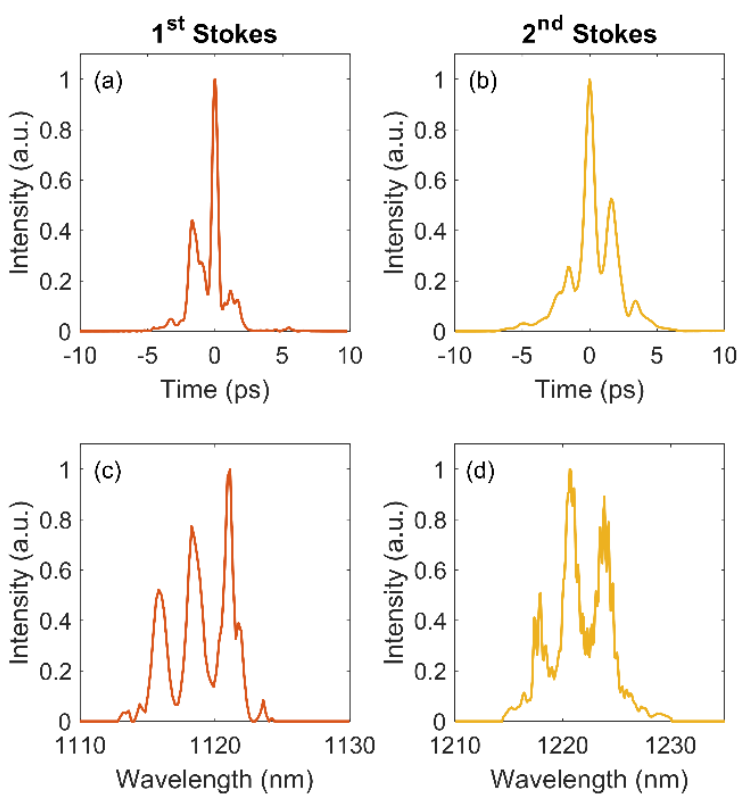

Fig. 6. Left column: (a) FROG-retrieved temporal intensity profile and (c) FROG-retrieved spectral intensity profile of the compressed $1^{\text {st }}$ Stokes at $93 \mu \mathrm{J}$ input energy; Right column: (b) FROG-retrieved temporal intensity profile and (d) FROG-retrieved spectral intensity profile of the compressed $2^{\text {nd }}$ Stokes at $126 \mu$ input energy.

Finally, the Stokes beams at the MPC output are temporally compressed using a pair of diffraction gratings in transmission, at an input energy corresponding to maximum efficiency for each line. The optimal compression point is searched by fine adjustment of the incident angle on the gratings and of the distance separating them. Compression of the $1^{\text {st }}$ Stokes beam is carried out using a pair of gratings with 1570 lines $/ \mathrm{mm}$ and yields a pulse duration of 520 fs at FWHM as shown in Fig. 6(a). The compressor throughput is $80 \%$. For the $2^{\text {nd }}$ Stokes beam, a pair of gratings with 1400 lines/mm is used, resulting in a duration of $800 \mathrm{fs}$ at FWHM, as shown in Fig. 6(b), with a compressor efficiency of $66 \%$. The obtained temporal pulse quality is mediocre, with large pedestals and side pulses. This can be traced back to the time-frequency structure evidenced by the spectrogram, showing a nonhomogeneous chirp rate, or equivalently a higher order spectral phase, that cannot be compensated by the gratings pair. We also notice that the obtained spectra for both the $1^{\text {st }}$ and $2^{\text {nd }}$ Stokes possess a peakstructure, as shown in Fig. 6(c) and (d), in agreement with the independently measured spatially-resolved spectrum shown in Fig. 3(d). This structure is likely related to the SPM/XPMinduced spectrogram shaping observed in Fig. 5.

In conclusion, we report the use of a solid-state material-based MPC to perform Raman frequency conversion. This allows efficient generation of the Stokes lines while preserving the beam quality due to the spatial filtering action provided by propagation inside the MPC. These setups could be used in other temporal regimes, e. g. with ps or ns pulses. Other efficient Raman materials such as diamond or molecular gases such as hydrogen could also be used to extend the possibilities in terms of target wavelength and input pulse energy. MPCs could also provide interesting capabilities: by carefully designing the MPC mirror coatings, it might be possible to stop the Raman cascade at a given Stokes line thereby optimizing the energy transfer towards this line. Finally, since Raman scattering is an inelastic process, scaling the average power can be limited by thermal effects $[17,18]$. In this MPC scheme, the thermal load is distributed to distinct spots at each pass through the Raman material, which could be advantageous. We therefore believe that Raman conversion is an interesting application of nonlinear MPCs.

Funding. Agence Nationale de la Recherche (ANR) (ANR-10-LABX0039-PALM).

Disclosures. The authors declare no conflicts of interest.

Data availability. Data underlying the results presented in this paper are not publicly available at this time but may be obtained from the authors upon reasonable request.

\section{REFERENCES}

1. R. Casula, J. Penttinen, M. Guina, A. J. Kemp, and J. E. Hastie, Optica 5, 1406-1413 (2018).

2. P. Mackonis, A. Petrulenas, A. M. Rodin, V. Girdauskas, and A. Michailovas, Opt. Lett. 45, 6627-6630 (2020).

3. G. Hilfer and C. R. Menyuk, J. Opt. Soc. Am. B 7, 739-749 (1990).

4. C. Jordan, K. A. Stankov, G. Marowsky and E. J. Canto-Said, Appl. Phys. B 59, 471-473 (1994).

5. N. Zhavoronkov, F. Noack, V. Petrov, V. P. Kalosha, and J. Herrmann, Opt. Lett. 26, 47-49 (2001).

6. F. B. Grigsby, P. Dong, and M. C. Downer, J. Opt. Soc. Am. B 25, 346350 (2008).

7. S. Zhou, T. Takamido, S. Imai, and F. Wise, Opt. Lett. 35, 2397-2399 (2010).

8. C. Vicario, M. Shalaby, A. Konyashchenko, L. Losev, and C. P. Hauri, Opt. Lett. 41, 4719-4722 (2016).

9. P. A. Carpeggiani, G. Coccia, G. Fan, E. Kaksis, A. Pugžlys, A. Baltuška, R. Piccoli, Y.-G. Jeong, A. Rovere, R. Morandotti, L. Razzari, B. E. Schmidt, A. A. Voronin, and A. M. Zheltikov, Optica 7, 1349-1354 (2020).

10. P. Cerny, H. Jelinkova, P. G. Zverev, T. T. Basiev, Prog. Quant. Electron. 28, 113-143 (2004).

11. V. A. Lisinetskii, V. A. Orlovich, H. Rhee, X. Wang, and H. J. Eichler, Appl. Phys. B. 91, 299-303 (2008).

12. J. Schulte, T. Sartorius, J.Weitenberg, A. Vernaleken, and Peter Russbueldt, Opt. Lett. 41, 4511-4514 (2016).

13. G. Jargot, N. Daher, L. Lavenu, X. Delen, N. Forget, M. Hanna, and P. Georges, Opt. Lett. 43, 5643-5646 (2018).

14. M. Ueffing, S. Reiger, M. Kaumanns, V. Pervak, M. Trubetskov, T. Nubbemeyer, and F. Krausz, Opt. Lett. 43, 2070-2073 (2018).

15. L. Lavenu, M. Natile, F. Guichard, Y. Zaouter, X. Delen, M. Hanna, E. Mottay, and P. Georges, Opt. Lett. 43, 2252-2255 (2018).

16. D. Herriott, H. Kogelnick, and R. Kompfner, Appl. Opt. 3(4), 523-526 (1964).

17. A. McKay, O. Kitzler, and R. P. Mildren, Opt. Express 22, 707-715 (2014).

18. M. Heinzig, G. Palma-Vega, B. Yildiz, T. Walbaum, T. Schreiber, and A. Tünnermann, Opt. Lett. 46, 1133-1136 (2021). 


\section{FULL TITLE REFERENCES}

1. R. Casula, J. Penttinen, M. Guina, A. J. Kemp, and J. E. Hastie, "Cascaded crystalline Raman lasers for extended wavelength coverage: continuous-wave, third-Stokes operation," Optica 5, 1406-1413 (2018).

2. P. Mackonis, A. Petrulenas, A. M. Rodin, V. Girdauskas, and A. Michailovas, "Two-stage transient stimulated Raman chirpedpulse amplification in KGd(WO4)2 with compression to $145 \mathrm{fs}$," Opt. Lett. 45, 6627-6630 (2020).

3. G. Hilfer and C. R. Menyuk, "Stimulated Raman scattering in the transient limit," J. Opt. Soc. Am. B 7, 739-749 (1990).

4. C. Jordan, K. A. Stankov, G. Marowsky and E. J. Canto-Said, "Efficient compression of femtosecond pulses by stimulated Raman scattering,"Appl. Phys. B 59, 471-473 (1994).

5. N. Zhavoronkov, F. Noack, V. Petrov, V. P. Kalosha, and J. Herrmann, "Chirped-pulse stimulated Raman scattering in barium nitrate with subsequent recompression," Opt. Lett. 26, 47-49 (2001).

6. F. B. Grigsby, P. Dong, and M. C. Downer, "Chirped-pulse Raman amplification for two-color, high-intensity laser experiments," J. Opt. Soc. Am. B 25, 346-350 (2008).

7. S. Zhou, T. Takamido, S. Imai, and F. Wise, "Exploitation of stimulated Raman scattering in short-pulse fiber amplifiers," Opt. Lett. 35, 2397-2399 (2010).

8. C. Vicario, M. Shalaby, A. Konyashchenko, L. Losev, and C. P. Hauri, "High-power femtosecond Raman frequency shifter," Opt. Lett. 41, 4719-4722 (2016).

9. P. A. Carpeggiani, G. Coccia, G. Fan, E. Kaksis, A. Pugžlys, A. Baltuška, R. Piccoli, Y.-G. Jeong, A. Rovere, R. Morandotti, L. Razzari, B. E. Schmidt, A. A. Voronin, and A. M. Zheltikov, "Extreme Raman red shift: ultrafast multimode nonlinear space-time dynamics, pulse compression, and broadly tunable frequency conversion," Optica 7, 1349-1354 (2020).

10. P. Cerny, H. Jelinkova, P. G. Zverev, T. T. Basiev, "Solid state lasers with Raman frequency conversion," Prog. Quant. Electron. 28, 113-143 (2004).

11. V. A. Lisinetskii, V. A. Orlovich, H. Rhee, X. Wang, and H. J. Eichler, "Efficient Raman amplification of low divergent radiation in barium nitrate crystal," Appl. Phys. B. 91, 299303 (2008).

12. J. Schulte, T. Sartorius, J.Weitenberg, A. Vernaleken, and Peter Russbueldt, "Nonlinear pulse compression in a multi-pass cell," Opt. Lett. 41, 4511-4514 (2016).

13. G. Jargot, N. Daher, L. Lavenu, X. Delen, N. Forget, M. Hanna, and P. Georges, "Self-compression in a multipass cell," Opt. Lett. 43, 5643-5646 (2018).

14. M. Ueffing, S. Reiger, M. Kaumanns, V. Pervak, M. Trubetskov, T. Nubbemeyer, and F. Krausz, "Nonlinear pulse compression in a gas-filled multipass cell," Opt. Lett. 43, 2070-2073 (2018).

15. L. Lavenu, M. Natile, F. Guichard, Y. Zaouter, X. Delen, M. Hanna, E. Mottay, and P. Georges, "Nonlinear pulse compression based on a gas-filled multipass cell," Opt. Lett. 43, 2252-2255 (2018).

16. D. Herriott, H. Kogelnick, and R. Kompfner, "Off-axis paths in spherical mirror interferometers," Appl. Opt. 3(4),523-526 (1964).

17. A. McKay, O. Kitzler, and R. P. Mildren, "High power tungstatecrystal Raman laser operating in the strong thermal lensing regime," Opt. Express 22, 707-715 (2014).

18. M. Heinzig, G. Palma-Vega, B. Yildiz, T. Walbaum, T. Schreiber, and A. Tünnermann, "Continuous-wave cascaded second Stokes diamond Raman laser at $1477 \mathrm{~nm}$," Opt. Lett. 46, 1133-1136 (2021). 